\title{
Some harvesting system transforming energy wastes of compressed air to electricity
}

\author{
Jacek S. Leszczynski ${ }^{1 *}$, Krzysztof Kastelik ${ }^{1}$, Ryszard Kaminski ${ }^{1}$, Bartłomiej Tomasiak ${ }^{1}$, \\ Dominik Grybos ${ }^{1}$, Milosz Olszewski ${ }^{1}$, Anna Plewa ${ }^{1}$, Pawel Mermer ${ }^{2}$, Roman Rygal ${ }^{3}$, \\ Marian Soinski ${ }^{3}$ \\ ${ }^{1}$ AGH University of Science and Technology, Faculty of Energy and Fuels, Department of Hydrogen \\ Energy, Al. Mickiewicza 30, 30-059 Krakow, Poland \\ ${ }^{2}$ Hafner Sp. J./O Czestochowa, ul. Główna 14 42-200 Czestochowa, Poland \\ ${ }^{3}$ Magneto Sp z o.o., ul. Odlewników 43, 42-200 Czestochowa, Poland
}

\begin{abstract}
Energy harvesting devices are self-powered systems which are associated with input ambient energy and convert such energy to electrical one. In this study, we focus on input ambient energy deriving from compressed air. Using arbitrary pneumatic machine, the compressed air after work - with its typical parameters as overpressure 6 bar or higher and variable volume flow - is exhausted to atmosphere. This generates energy losses. We have constructed some device which transforms the loss of compressed air energy to electricity. It illustrates the principle of action very well and does not perturb operation of pneumatic machines being source of energy losses.
\end{abstract}

\section{Introduction}

Alternative energy generation systems are rapidly expanding and being interconnected to distributed transmission networks (grid-on) or supply internal networks (grid-off). At present, one observes development in electrical energy storage technologies [1] and their application potential in power systems. However, it should be noted huge amount of energy losses at global scale. Thus, there is a plenty of room to recover the energy loss and to reproduce electricity. Within this background, energy harvesters [2] are a booming business at the level of watts to kilowatts. By name, energy harvesting devices [3] are self-powered systems which are associated with input ambient energy and its conversion to electrical energy. However, classical input ambient energies include heat (thermoelectric modules), light (photovoltaic cells), radiation (rectifying antennas) and vibration (piezoelectric, electro-magnetic, electro-static transducers).

In this study, we focus on input ambient energy deriving from compressed air. There is huge amount of systems, where primary air makes work due to compression, is exhausted from the system. Such process one observes in food industry - when packing or other machines are used, automotive industry - where pneumatic robots are applied, mechanical industry - where pneumatic presses or pneumatic motors are used, transport industry -

\footnotetext{
* Corresponding author: jale@agh.edu.pl
} 
where compressed air is necessary to open or close doors, etc. Moreover, one can produce compressed air from other phenomena, such as: frequently inhibiting/stopping vehicles, elevators which are falling down gravitationally or doors which are opening and closing very frequently. Generally speaking, production of compressed air costs input energy. After work, the compressed air, with typical parameters as overpressure 6 bar or higher and variable volume flow, is exhausted to atmosphere. This generates energy loss. Here we focus on such energy losses. We have constructed some device, which transforms the loss of compressed air energy - exhausted to atmosphere - to electricity.

\section{State of the art and test rig}

Our idea is restricted and patented in Polish Patent Office [4]. Fig. 1 illustrates scheme of installation which is able to transform any energy losses of compressed air to electrical energy.

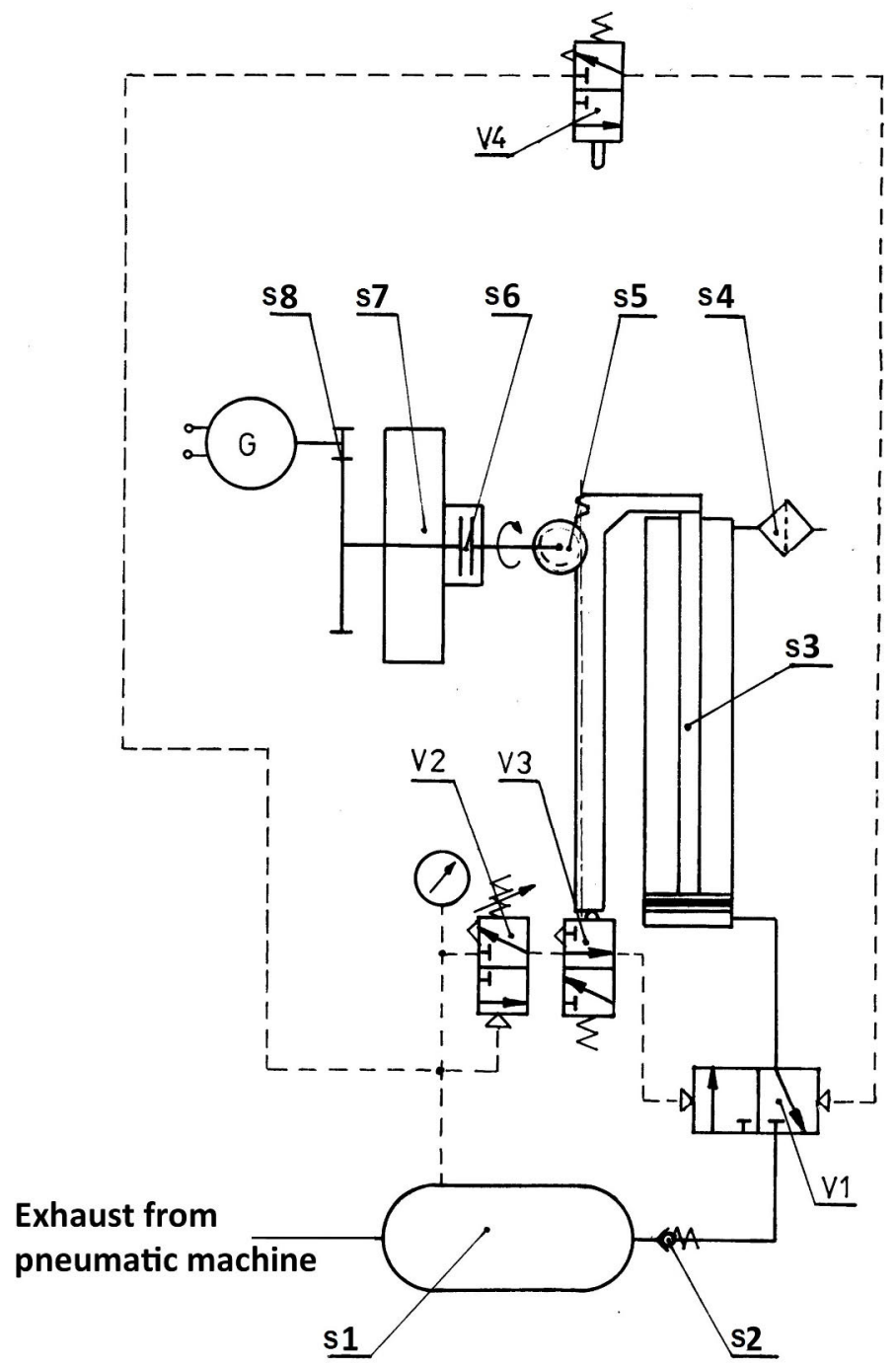

Fig. 1. Scheme illustrates energy recovery, according to our pending patent [4]. 
We look into energy of compressed air exhausting to atmosphere which is storing in some air tank (S1). Control valve (V1) is double controlled by valve (V2) and control valve (V4). When overpressure in tank (S1) riches control overpressure manually controlled from range 0.5 bar to 1.5 bar then, through one directional valve (S2), control valve (V1) is opening and low-compressed air drives cylinder (S3) automatically. Cylinder (S3) goes up running a kinematic pair - rack gear (S5). The kinematic pair drives power generator (G) through system of gears (S7) and (S8) with unidirectional clutch (S6). When cylinder (S3) hits high position then control valve (V4) switches automatically control valve (V1) and cylinder (S3) is falling down due to gravity till to lower positon determined by control valve (V3). Above description illustrates one cycle of operation. Next cycle starts and stops automatically being dependent on overall volume of tank (S3), the speed of compressed air filling tank (S3) and manually adjustable the air overpressure in the tank.

According to idea illustrated by Fig. 1 we are able to construct a test rig. Using $\mathrm{CAD} / \mathrm{CAM}$ technology we have elaborated the virtual model of our harvester. The model is presented by Fig. 2.

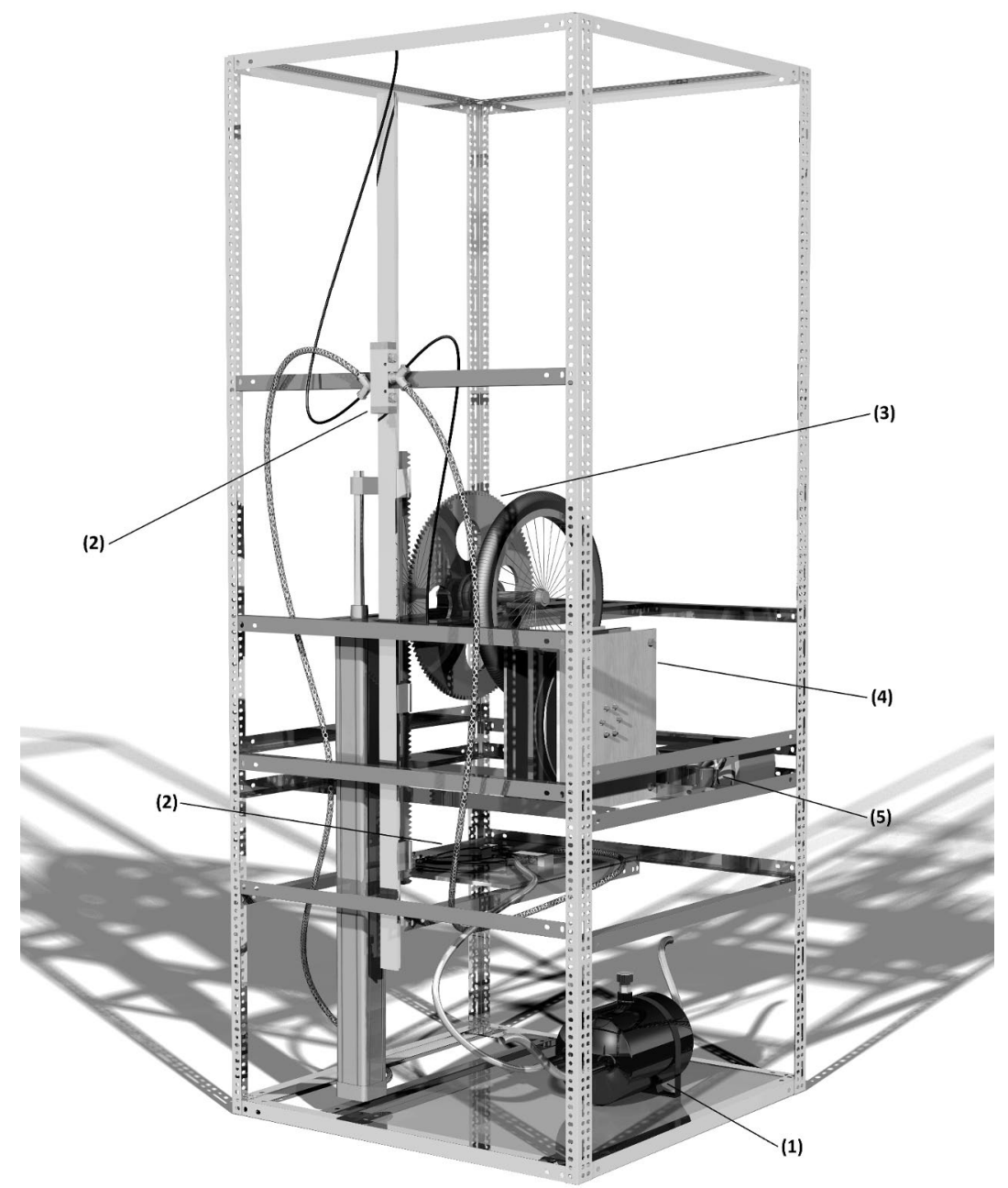

Fig. 2. Virtual construction of energy harvester: 1) overpressure tank; 2) pressure management system; 3) gears; 4) low speed power generator; 5) battery management system. 
One may distinguish main components of the energy harvester as: overpressure tank (1), pressure management system (2), system of friction and rack gears (3), low speed power generator (4) and battery management system (5).

In order to demonstrate how our idea operates in practice we have constructed the test ring in semi-industrial scale. Fig. 3 shows our construction.

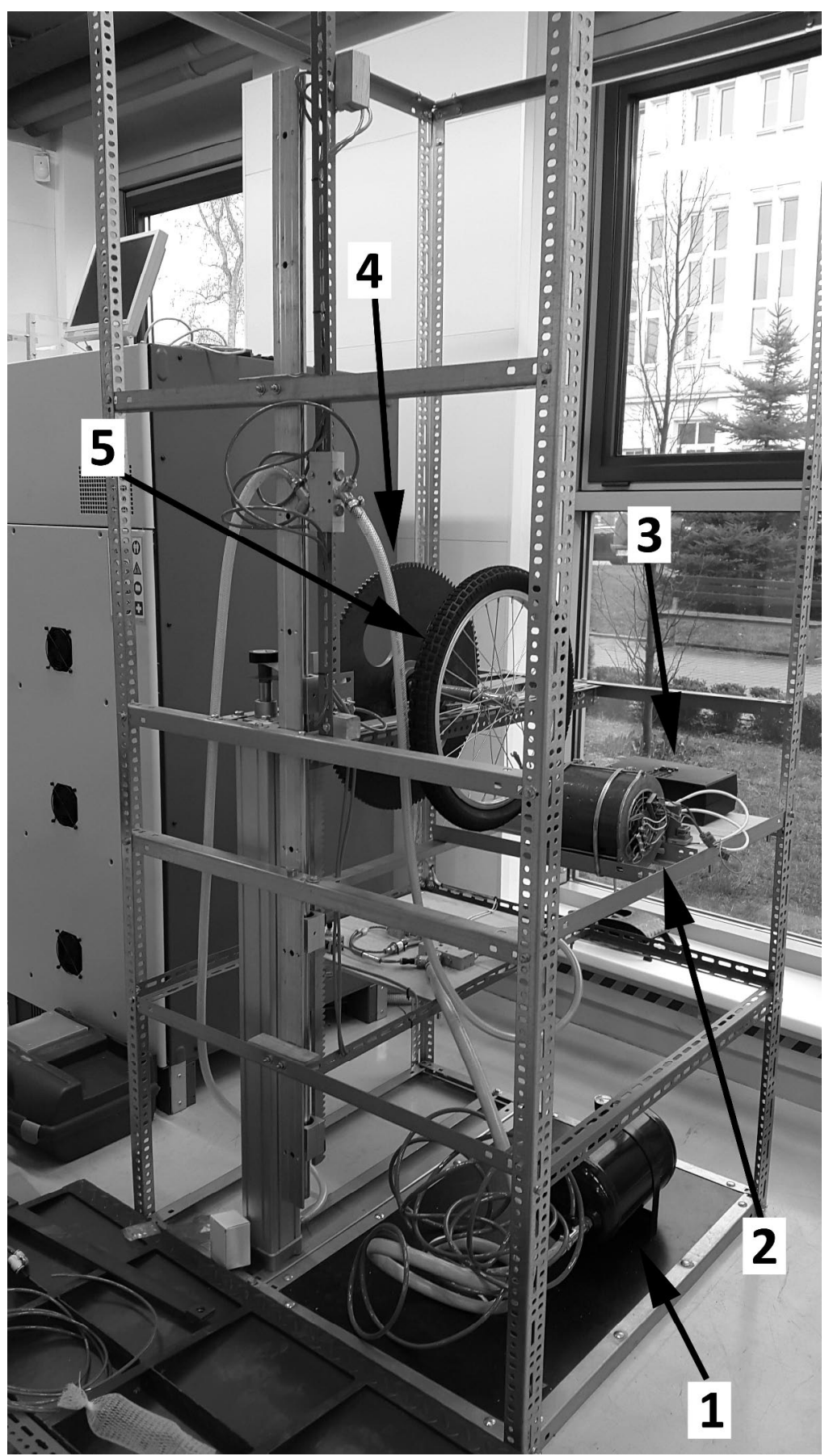

Fig. 3. Test rig in semi-industrial scale. 
We mounted air tank (1) - with volume $V_{z}=10 \mathrm{dm}^{3}$, low-speed power generator (2) which generates $500 \mathrm{~W}$ in $400 \mathrm{obr} / \mathrm{min}$, battery management system (3), system of rack gearwheel (4) with $d_{1}=0.5 \mathrm{~m}$ and length of rack $l_{r}=1 \mathrm{~m}$, system of friction gear $d_{2}=0.5 \mathrm{~m}$, $d_{3}=0.02 \mathrm{~m}$. We applied the cylinder with the following technical data: $d_{s}=0,08 \mathrm{~m}, l_{c}=1 \mathrm{~m}$. Figures 4 and 5 present overpressure control system and battery management system in more details.

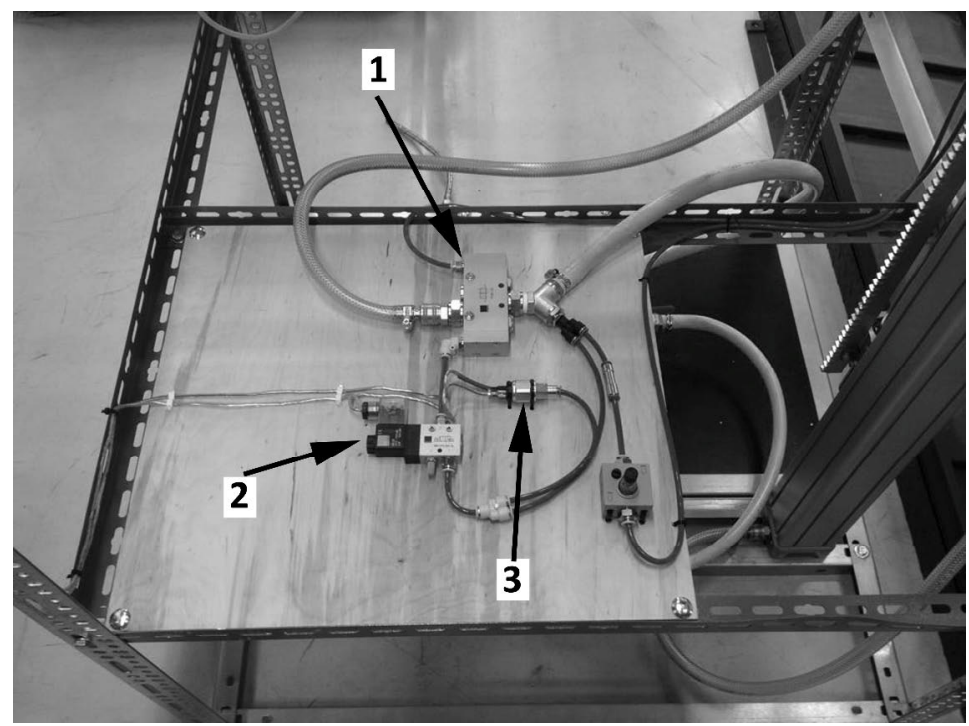

Fig. 4. Photograph of overpressure control unit.

The main components of the overpressure control unit are the following:

- $\quad 3 / 2$ control valve (1) of $1 / 4$ inch which is normally open and has spring return cylinder,

- control valve (2) of $1 / 8$ inch which is normally close and is controlled by electromagnetic coil,

- $\quad$ pressure sensor (3) controls the valve (1) by using the valve (2).

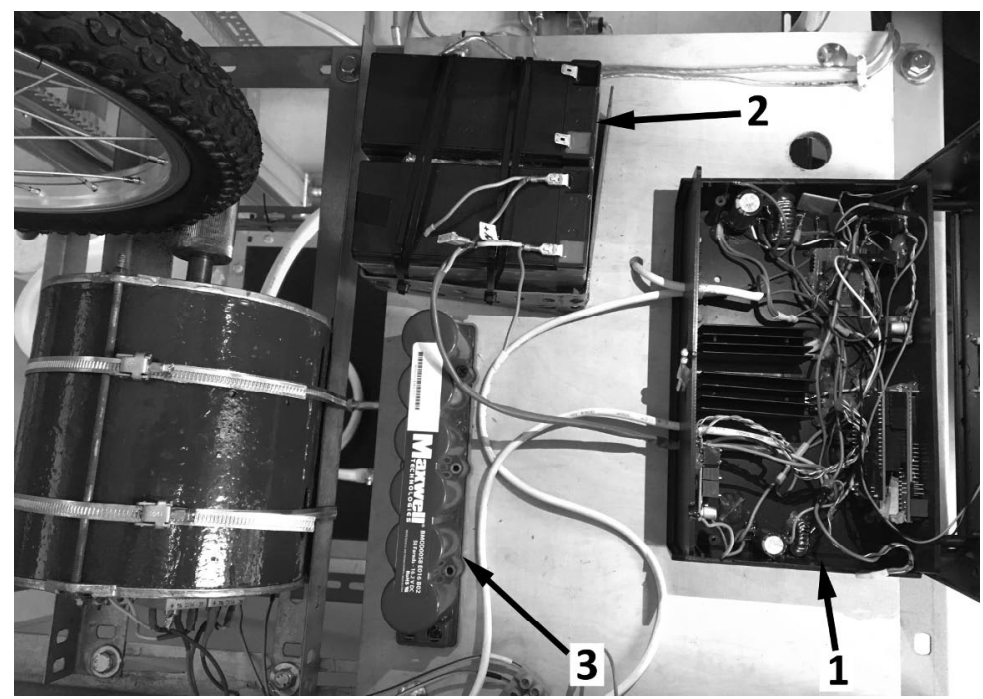

Fig. 5. Photograph of battery management system [5]. 
The main components of the battery management system are the following:

- electronic control unit composes from one AC/DC and two DC/DC transformers which transform arbitrary voltage level to $12 \mathrm{~V} \mathrm{DC}$,

- bank of two batteries with rechargeable battery capacity $C_{a}=7.2 \mathrm{Ah}$ and operating voltage $U_{a}=12 \mathrm{~V} \mathrm{DC}$,

- bank of supercapacitors with maximum $U_{c}=16 \mathrm{~V}$ DC operating voltage, rechargeable supercapacitors capacity $C_{c}=58 \mathrm{~F}$.

Using the technology demonstrator, as presented by Fig. 1 completely, we are able to perform several tests in order to assess how our system operates in real working environment.

\section{Technological tests}

Before we begin full technological tests, we try to investigate how our power generator works. In this case we use FEMM code [6] that to simulate the generator behaviour in order to assess its mechanical constructions. We assume idle operating state of the generator that to observe magnetic flux density. Fig. 6 shows distribution of magnetic flux density in the kinematic pair rotor-stator.
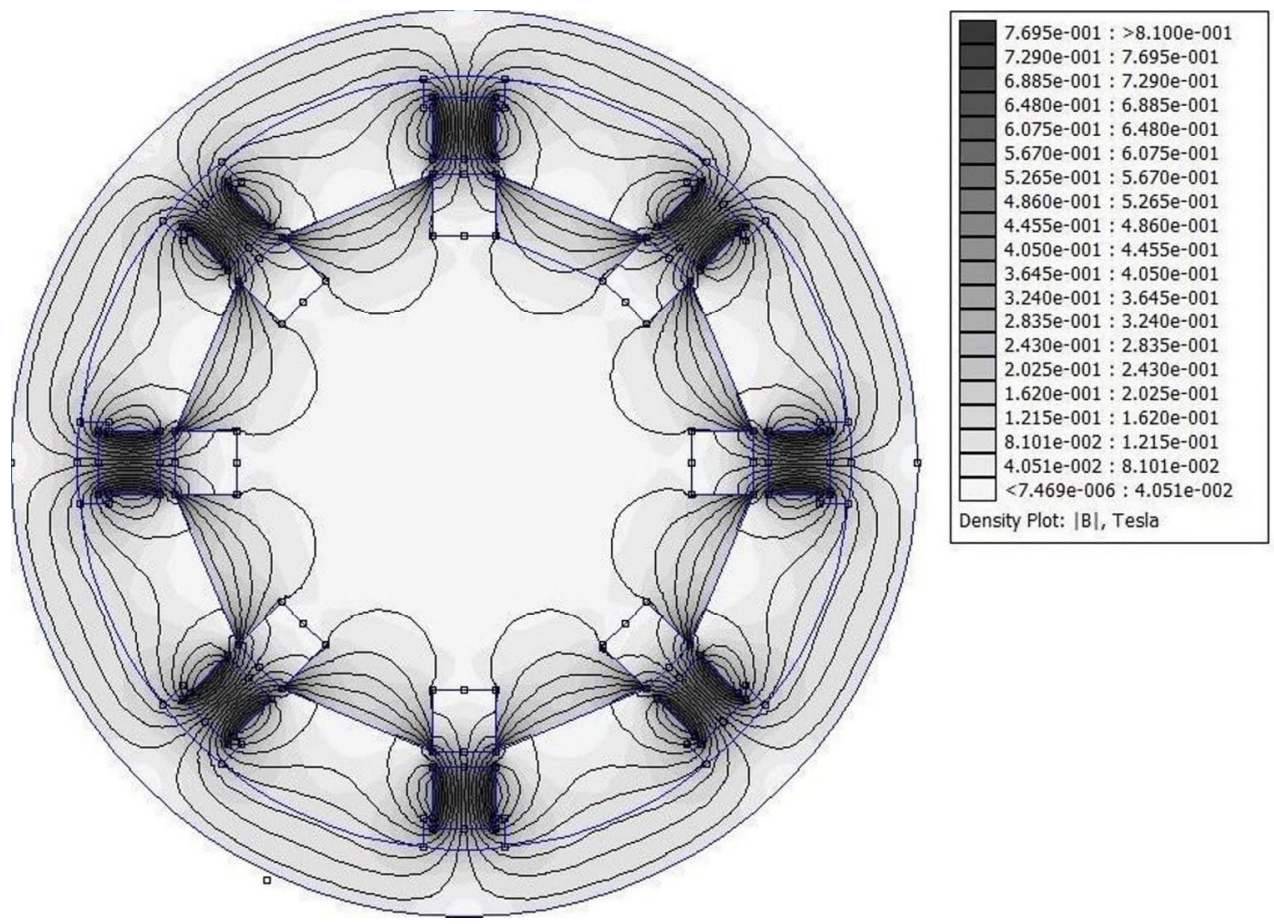

Fig. 6. Simulation of magnetic flux density has been done by FEMM [6], cross section, idle state of the power generator.

In simulating conditions the generator rotates with the rotational speed $300 \mathrm{r} / \mathrm{min}$. We observe concentration of the magnetic flux near neodymium magnets and magnetic field lines closes. This means that there is no big dissipation of magnetic energy. Next we would like to estimate how the magnetic field is shielded. Fig. 7 presents magnetic flux density distribution in horizontal section. 


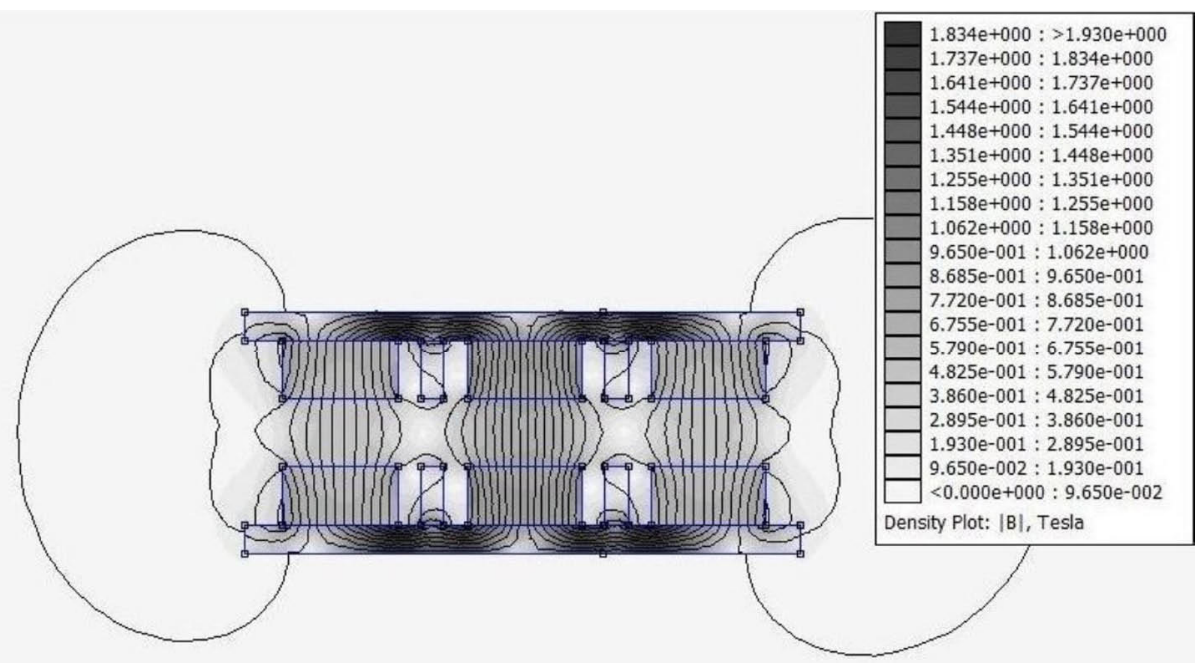

Fig. 7. Simulation of magnetic flux density has been done by FEMM [6], horizontal section, idle state of the power generator.

We are able to assess the thickness of rotating plate in order to find minimal thickness being robust for magnetic saturation. Here we establish $h=5 \mathrm{~mm}$ thickness of rotating steel plate. One notices closed lines of magnetic flux. This indicates no magnetic dispersion and therefore the minimal value of plate thickness fulfils its function, i.e. the magnetic shield.

Next we analyse spatial distribution of the magnetic flux density in the piece of low speed generator. Fig. 8 shows such situation.

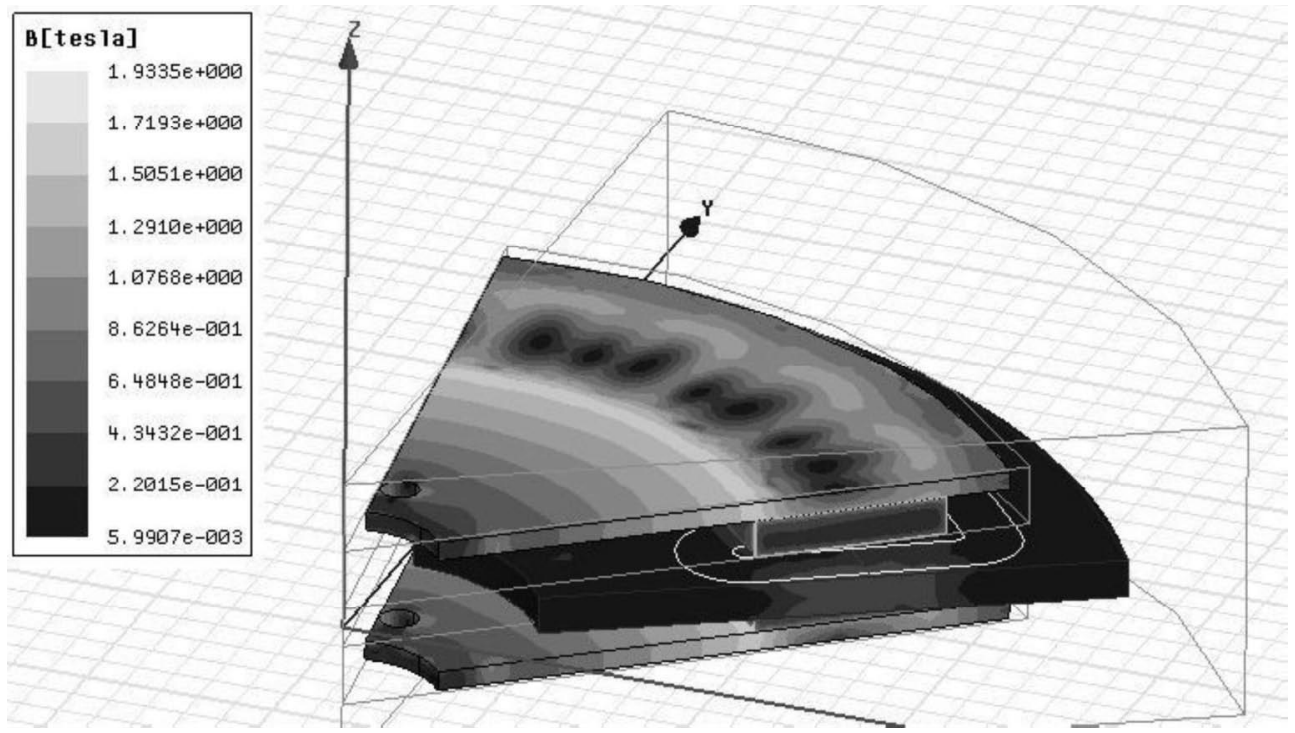

Fig. 8. Simulation of magnetic flux density has been done by FEMM [6], spatial-view, idle state of the power generator.

We observe right distribution of the magnetic flux density in quarter of the kinematic pair rotor-stator. Values of magnetic fluxes are not high and not exceed saturation. This means the distribution of magnetic fluxes guarantees right operation of the power generator. To 
summarise, we have analysed the power generator computationally. There is no obstacle in the proposed construction and therefore the power generator is ready for operation.

In order to proof how our harvester operates, we performed several industrial tests. We connected our unit throughout the tank to a pneumatic machine, to the valve where compressed air was exhausted to atmosphere. Notice oscillations of overpressure of compressed air in the pneumatic machine around 6 bar. After work, the compressed air through a system of valves is exhausted to atmosphere. Our tank with volume $V_{z}=10 \mathrm{dm}^{3}$ fills quite fast to the control overpressure 1.5 bar. After the control overpressure is reached, the control valve opens and then the stored compressed air in the tank runs the cylinder. In the tank we notice decompression till to 0.75 bar. The cylinder uses $2250 \mathrm{dm}^{3} / \mathrm{h}$ volumetric flow of the compressed air in one cycle of its operation. Through the system of gears, the cylinder runs the power generator. Table 1 shows values of the power generator registered in one cycle of cylinder operation - from the bottom position to the top position.

Table 1. Values of power generator registered under idle state.

\begin{tabular}{|c|r|r|}
\hline Measurement no. & \multicolumn{1}{c|}{$\begin{array}{c}\boldsymbol{U}_{\boldsymbol{o}} \\
{[\mathbf{V}]}\end{array}$} & $\begin{array}{c}\boldsymbol{n}_{\boldsymbol{o}} \\
{[\mathbf{r} / \mathbf{m i n}]}\end{array}$ \\
\hline 1 & 70 & 32 \\
\hline 2 & 98 & 202 \\
\hline 3 & 100 & 214 \\
\hline 4 & 80 & 93 \\
\hline 5 & 99 & 208 \\
\hline
\end{tabular}

Above table presents voltage and rotating speed values which were registered under the idle state of power generator. This means no external load has been noted. Mean values are the following: $\bar{U}_{0}=89 \mathrm{~V}$ and $\bar{n}_{0}=150 \mathrm{r} / \mathrm{min}$.

Next we would like to observe what happens when we upload the generator. Fig. 9 and Fig. 10 present two scenarios of the operating generator under different loads.

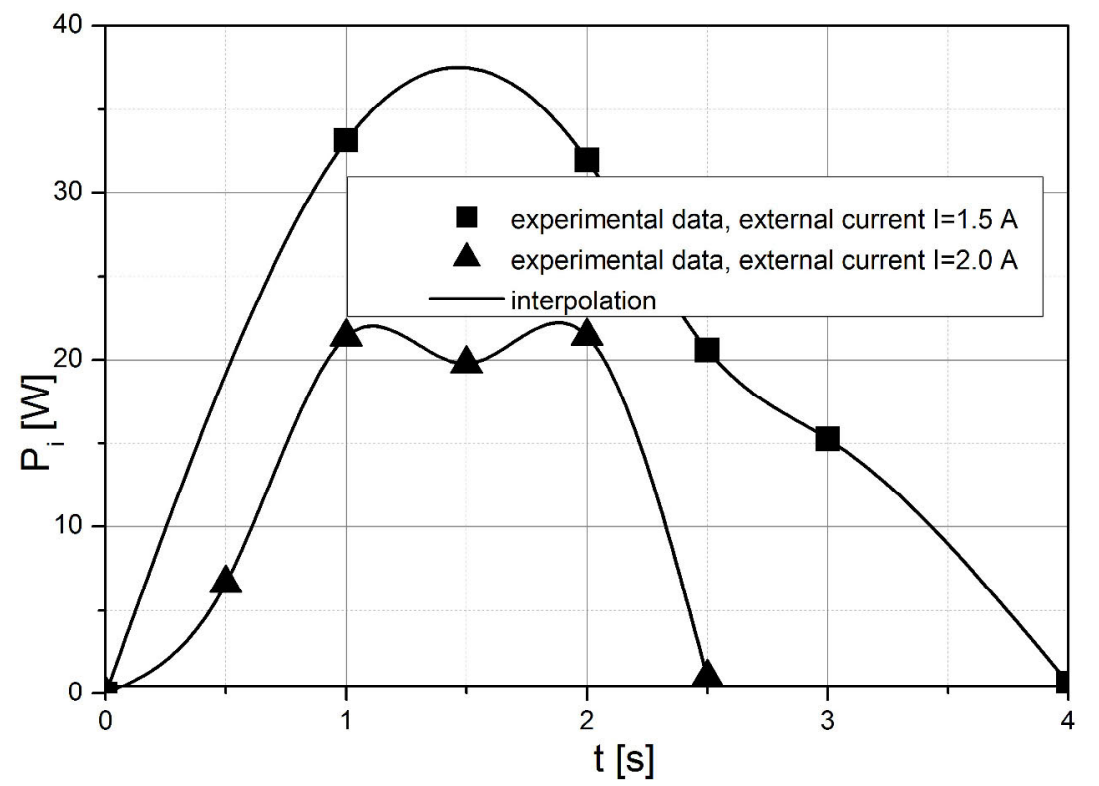

Fig. 9. Power produced in generator over time registered under one motion of cylinder in two load states. 


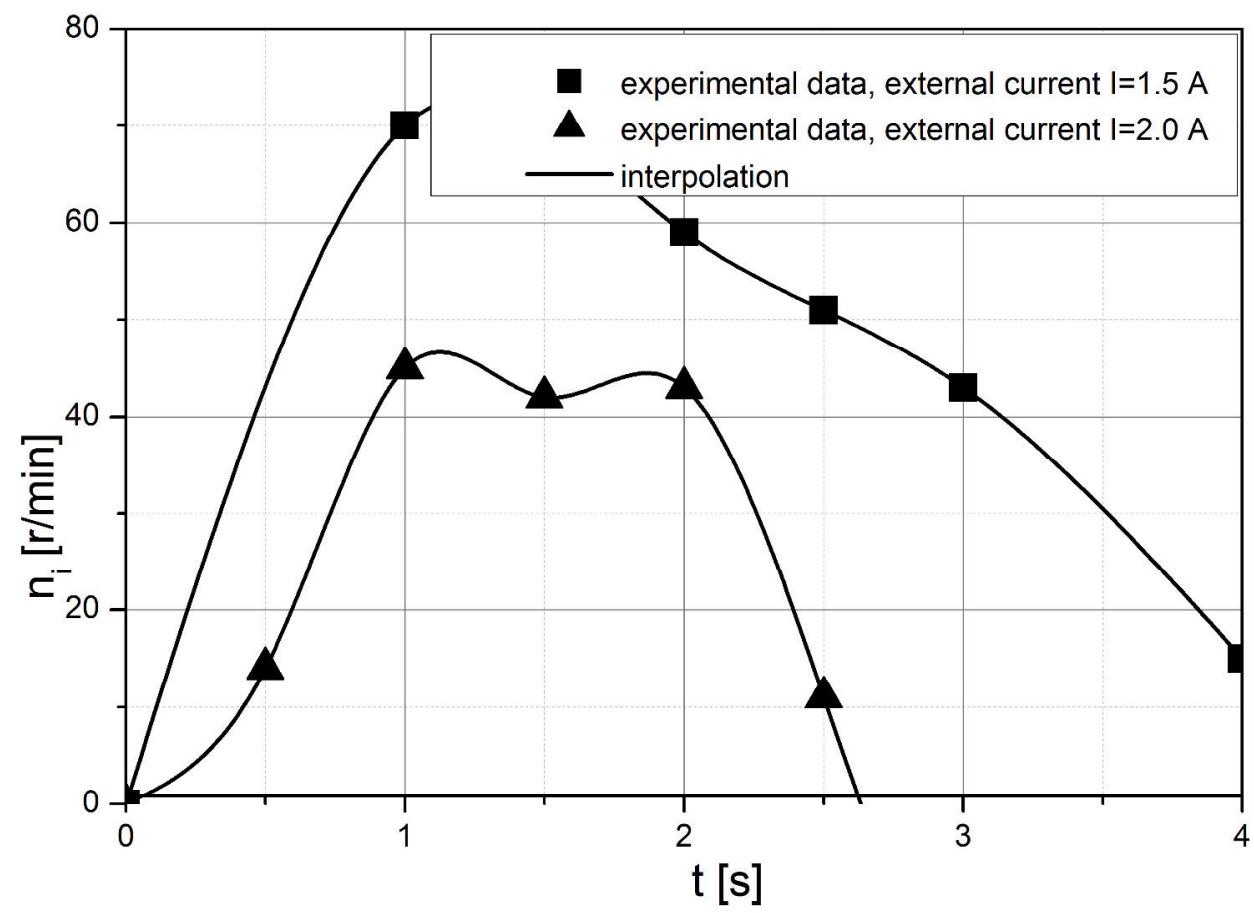

Fig. 10. Rotational speed of generator over time registered under one motion of cylinder in two load states.

In one cycle of cylinder operation we use $2250 \mathrm{dm}^{3} / \mathrm{h}$ volumetric flow of the compressed air. The compressed air during its work into the cylinder is decompressed dynamically. The cylinder starts go up from overpressure 1.5 bar and ends the cycle to 0.75 bar. Duration over time the adiabatic decompression process varies from equals around $1.5 \mathrm{~s}$, when we load the power generator. Time for the idle return of the cylinder, from the upper position to the lower one under gravity only, equals to $2.5 \mathrm{~s}$. Here we noted average velocities of the piston motion around $w_{l}=0.67 \mathrm{~m} / \mathrm{s}$ in the cylinder operating state and velocity $w_{2}=0,4 \mathrm{~m} / \mathrm{s}$ in the cylinder idle return. In order to assess how effective is our energy harvester we perform two technological tests when the power generator was loaded in some resistance way and we noted the following currents $1.5 \mathrm{~A}$ for test no. 1 and $2.0 \mathrm{~A}$ for test no. 2. Fig. 9 shows changes of power over time which was registered on the generator ends. One notes more power registered over time for low load resistance, when current was $1.5 \mathrm{~A}$. This situation was explained in Fig. 10 where rotational speed over time for the two above cases was registered. Here we observe higher values of the rotational speed when resistance load was $1.5 \mathrm{~A}$. To conclude we have noted that the power generator is not sufficient to work in low rotational speeds. This means we need to elaborate a novel model of the power generator featuring low internal resistance and therefore being more efficient in the range of low rotational speeds. However, our industrial test does not perturb normal operation of the pneumatic machine being involved that to recover the loss of energy of compressed air.

\section{Concluding remarks}

In this study, we focus on input ambient energy deriving from compressed air. Using arbitrary pneumatic machine we add our harvester to values where the compressed air, with its typical overpressure 6 bar or higher, after work is exhausted to atmosphere. Here we constructed the device which transforms the loss of compressed air energy to electricity. 
Additional process of energy transformation does not perturb the primary operation of pneumatic machine under condition that maximal value of overpressure in the tank does not exceed 1.5 bar. Our energy harvester is not efficient and not perfect device. At present the harvester illustrates the principle of action very well and does not perturb operation of pneumatic machines being source of energy losses. In further researches we need to elaborate a mathematical model which will illustrate the dynamics of compressed air and the dynamics of all devices involved in the energy harvester. Simple technological tests confirm that the power generator used in the harvester features by high internal resistance and therefore is not adequate to operate in its low rotational speeds.

\section{References}

1. X. Luo, J. Wang, M. Dooner, J. Clarke, Overview of current development in electrical energy storage technologies and the application potential in power system operation, Applied Energy 137, 511-536 (2015)

2. P. Harrop, An Introduction to Energy Harvesting (IDTechEx, www.IDTechEx.com, 2009)

3. P. Spices, L. Mateu, M. Pollak, Handbook of Energy Harvesting Power Supplies and Applications (Pan Stanford Publishing Pte Ltd., Singapore, 2015)

4. J.S. Leszczynski, K. Kastelik, R. Kaminski, et al., A device for processing pulses of gravitational energy into electricity, patent pending No. P.414139, Polish Patent Office, Warsaw, Poland (2016)

5. B. Tomasik, A. Plewa, J.S. Leszczynski, Electronic system for pulse energy storage (Bulletin of the Polish Hydrogen and Fuel Cells Association 9, 14th Symposium on Fast ionic conductors : 22-25.09.2015, Bialka Tatrzanska, Poland, 58, 2015)

6. D. Meeker, Finite Element Method Magnetics (Version 4.2, User's Manual, October 25, 2015) 